\title{
Endoscopic ultrasound-guided fine-needle biopsy is superior to fine-needle aspiration in assessing pancreatic neuroendocrine tumors
}

다(1) $(9)$

\begin{abstract}
Authors Kofi W. Oppong ${ }^{1}$

Institutions

1 HPB endoscopy unit, Newcastle Upon Tyne Teaching Hospitals Foundation Trust, Newcastle Upon Tyne, UK

2 HPB Surgical unit, Newcastle Upon Tyne Teaching Hospitals Foundation Trust, Newcastle Upon Tyne, UK

3 Department of Cellular Pathology, Newcastle Upon Tyne Teaching Hospitals Foundation Trust, Newcastle Upon Tyne, UK
\end{abstract}

John S Leeds ${ }^{1}$, Manu K. Nayar ${ }^{1}$, Noor L.H. Bekkali ${ }^{1}$, Colin H. Wilson², Sarah J. Johnson ${ }^{3}$, Beate Haugk ${ }^{3}$, Antony Darne ${ }^{3}$,

submitted 2.4.2019

accepted after revision 24.6.2019

Bibliography

DOI https://doi.org/10.1055/a-0990-9611 |

Endoscopy International Open 2019; 07: E1281-E1287

(c) Georg Thieme Verlag KG Stuttgart · New York

elSSN 2196-9736

Corresponding author

Dr. J.S. Leeds, HPB endoscopy unit, Freeman Hospital, Freeman Road, Newcastle Upon Tyne, NE7 7DN, UK

Fax: + 01224559959

john.leeds@nuth.nhs.uk

\section{ABSTRACT}

Background and study aims Pancreatic neuroendocrine tumors (PanNETs) outcomes are dependent upon grading by Ki67. This study compared endoscopic ultrasound-guid- ed fine-needle biopsy (EUS-FNB) to fine-needle aspiration (FNA) in assessing PanNETs.

Patients and methods All pancreatic histology for PanNET between January 2009 and June 2017 was included if EUS sampling was performed prior to surgical resection. Ki67 and grade from FNA and FNB samples was compared to surgical histology using correlation coefficient and kappa values. Subgroup analysis was performed for purely solid lesions, lesions $<2 \mathrm{~cm}$ and FNB needle type. which 57 underwent surgical resection. Thirty-five lesions underwent FNA and 26 FNB (4 had both) confirming PanNET. 23/ of 35 FNA samples reported Ki67/grading compared to all 26 FNB samples $(P=0.0006)$. Compared to surgical histology, Ki67 on FNA correlated poorly overall $(r=-0.08)$, in solid lesions $(r=-0.102)$ and lesions $<2 \mathrm{~cm}(r=-0.149)$ whereas FNB correlated moderately overall $(r=0.65)$, in solid lesions $(r=0.64)$ and lesions $<2 \mathrm{~cm}(r=0.61)$. Tumor grade showed poor agreement (kappa) with FNA overall (0.026), in solid lesions (0.044) and lesions < $2 \mathrm{~cm}(0.00)$ whereas FNB showed moderate-good agreement overall $(0.474)$, in solid lesions (0.58) and lesions $<2 \mathrm{~cm}(0.745)$. Fork-tip FNB needles Ki67 showed strong correlation with surgical histology $(r=0.788)$ compared to reverse bevel FNB needles $(r=0.521)$. Both FNB needles showed moderate agreement with tumor grade.

Conclusion FNB samples were significantly more likely than FNA to provide adequate material for Ki67/grading and showed a closer match to surgical histology. FNB needle types require prospective investigation.
Results One hundred sixity-four patients had PanNET of

\section{Introduction}

Pancreatic neuroendocrine tumors (PanNETs) are rare and account for between $1 \%$ and $5 \%$ of pancreatic mass lesions [1, 2]. Most PanNETs are low grade and run an indolent course but recent data suggest a significant increase in incidence $[1,3]$. PanNETs can be classified in a number of ways including functional status and may present with clinical symptoms dependent on the hormone being secreted; however, about $40 \%$ of
PanNETs are non-functional [2]. Management and subsequent prognosis of PanNETs is guided by the Ki67 proliferative index (PI) which has been validated in a number of studies and incorporated in to international guidelines [4-7]. This is particularly important in patients with non-functioning PanNETs less than $2 \mathrm{~cm}$ as the risk:benefit equation of surgery versus observation is harder to judge. In these circumstances, Ki67 PI is crucial in guiding discussion between clinicians and patients as to the most suitable management plan [5]. 
Endoscopic ultrasound-guided fine-needle aspiration (EUSFNA) has become the procedure of choice for obtaining tissue from pancreatic lesions. With respect to PanNETs, EUS-FNA has been used to provide cytological material for pathological analysis for many years [8]. Previous studies have shown that EUSFNA is useful in diagnosing PanNET but the ability to provide an estimate of Ki67 PI appears variable [9-13]. This may be due to a number of factors including lack of cellular cohesion in cytological preparations and adequacy of material for sufficient staining of relevant cells $[11,13]$. Several studies have examined the role of EUS-FNA in patients with PanNETs but there are important limitations in each of them [8-15]. The main limitation in most of these studies is the small numbers of patients included, retrospective nature and lack of a gold standard used for comparison i.e. surgery $[10,11,13,14,16,17]$. EUS-FNA appears to underestimate Ki67 PI, leading to undergrading of PanNETs with some studies showing EUS-FNA samples labelled as grade 1 being upgraded to grade 2 on surgical resection histology $[16,17]$. The "holy grail” would be to obtain histological quality samples to provide the pathologist with sufficient material to make a firm diagnosis of PanNET, accurately grade the lesion, and subtype using immunohistochemistry. Recently, EUS-guided fine-needle biopsy (EUS-FNB) has become available with several needle types designed to take core samples and preserve tissue architecture for histological assessment [18-20]. Some studies have shown improved accuracy for tissue sampling via EUS with some core needle types [18, 20] although a recent meta-analysis showed no improvement in sample adequacy or diagnostic accuracy for one type of core needle [21]. Despite this there are few studies comparing EUS-FNA with EUS-FNB particularly in circumstances where additional information would be crucial to patient management such as those with suspected PanNETs $<2 \mathrm{~cm}$. A pilot study showed fewer passes were required for diagnostic material when using an FNB needle compared to an FNA needle, however, no assessment was made of the ability to determine Ki67 $\mathrm{PI}$ and grade and no gold standard was used [22]. The aim of this study was to assess whether $\mathrm{Ki67} \mathrm{PI}$ and grading can be more accurately determined using FNB compared to FNA using surgical excision histology as the gold standard. Subgroup analysis of different needle types was also performed.

\section{Patients and methods}

The Freeman Hospital is a tertiary referral center for hepatobiliary and pancreatic diseases in the North East region of England providing services to approximately 3.5 million people. EUS is provided by three experienced pancreaticobiliary endosonographers (JSL, MKN, KWO) with a minimum of 3000 EUS performed individually.

Retrospective analysis of all pancreatic pathology reports for neuroendocrine tumors was performed for the period January 2009 to June 2017. The pathology database was searched using SNOMED codes for all surgical resections of PanNET lesions that also had preoperative EUS-guided sampling. Patient demographics, lesion size, and location were noted. FNA and FNB reports were examined and Ki67 PI and grade recorded. Surgical histology reports were examined and time from EUS to surgery, operation performed, TNM stage, Ki67 and grade recorded and compared using correlation coefficient and proportional analysis.

All EUS procedures were undertaken after obtaining informed patient consent. Hitachi EUB-7500 or Preirus US workstations (Hitachi Medical Systems, Wellingborough, UK) and Pentax linear echoendoscopes (Pentax, Slough, UK) were used to perform EUS.Sampling of the lesion was performed under real-time EUS guidance using the techniques described hereafter. All procedures were performed using the slow pull technique followed by $10 \mathrm{~mL}$ of suction during the passes and fanning of the needle throughout the lesion to optimize tissue acquisition. The number of passes into the lesion was determined by the individual endosonographer but a minimum of three passes was the target during the study period. Rapid On Site Evaluation (ROSE) was available during the study period but only applied to the FNA samples and we have previously shown no increase in diagnostic yield in an established EUS service [23].

\section{EUS-FNA technique}

FNA was performed using either 22- or 25-gauge needles (Cook Medical, Limerick, Ireland) and tissue from each pass expressed onto glass slides to create smear slides which were air-dried and subsequently stained with Giemsa-based stains. The remaining tissue was expressed into cytofix red solution (BD Surepath, Bioscience Healthcare, Nottingham, UK) and used for Surepath Liquid Based Cytology (LBC). Any macroscopically intact tissue fragments were processed as a cell block and stained with hematoxylin and eosin. All slides were analyzed by experienced cytopathologists. Cytological diagnosis of well-differentiated neuroendocrine tumor was based on standard features (such as mostly dispersed cell population with eccentric cytoplasm, smooth nuclear outline with salt-and-pepper chromatin, inconspicuous nucleoli and naked nuclei). Immunocytochemistry was performed on the LBC or cell block (cell block used in preference if contained enough tumor cells), with the neuroendocrine markers synaptophysin, chromogranin and CD56, plus Ki67 when sufficient material was available. Ki67 was assessed by estimation or cell counting and tumors were given a provisional grade.

\section{EUS-FNB technique}

EUS-FNB was performed using either ProCore needles (Cook Medical, Limerick, Ireland) or Sharkcore needles (Covidien/ Medtronic, Whiteley, UK). All passes were placed in one container of neutral buffered formalin which was sent for standard histopathological processing and assessment was by experienced histopathologists. Diagnosis of well differentiated neuroendocrine tumor was made on the basis of histomorphological characteristics (i.e. presence of tumor tissue fragments of loosely cohesive, fairly monotonous cells with regular nuclei and homogenous/finely stippled chromatin) and immunohistochemical confirmation through positive staining for pancytokeratin, synaptophysin and chromogranin and/or CD56. In a proportion of cases specific hormones and cytokeratin 19 were 
performed to provide additional prognostic and management information. All cases were stained for Ki67 to assess proliferation index and inform histological grade. Ki67 PI was determined by direct cell count under high magnification with or without aid of a manual cell counter.

\section{Outcome measures}

The primary objective was to determine whether EUS-FNA or FNB samples correlate with surgical resection histology samples with particular reference to Ki67 PI and grading in all PanNETs.

The secondary objective was to assess the degree of mismatch between EUS-guided sampling and surgical resection histology with reference to under- and over-grading. Subgroup analysis on purely solid PanNETs and of the different FNB needle types was also performed.

\section{Ki67 proliferation index and grading}

Grade of PanNETs is based on mitotic activity and Ki67 PI. The WHO classification 2010 provides a three-tier system defined as follows: grade 1:<2 mitoses/10 hpf $\left(2 \mathrm{~mm}^{2}\right), \mathrm{Ki} 67<2 \%$; grade 2: 2 to 20 mitoses/10 hpf ( $\left.2 \mathrm{~mm}^{2}\right)$, Ki67 3\% to $20 \%$; grade 3:> 20 mitoses/10 hpf ( $\left.2 \mathrm{~mm}^{2}\right), \mathrm{Ki67}>20 \%$ [22]. The boundary between grade 1 and 2 PanNETs was changed in the UK in 2012: grade 1: $<2$ mitoses/10 hpf $\left(2 \mathrm{~mm}^{2}\right)$, Ki67 $\leq 5 \%$; grade 2: 2 to 20 mitoses/10 hpf $\left(2 \mathrm{~mm}^{2}\right)$, Ki67>5\% to $20 \%$ [24]. However, EUSguided sampling and surgical histology samples were assessed using the grading system used during the relevant clinical period. Therefore, the threshold for the grade was compared at the relevant time and not reallocated according to the most current guidelines.

\section{Statistical analysis}

Parametric data were described using mean and standard deviation whereas non-parametric data were described using median and interquartile range. Proportional analysis was performed using either Chi-square or Fishers exact test. To compare Ki67 PI in EUS-guided samples and surgical resection histology, correlation coefficients were calculated using Pearson's r. Pearson's $r$ values were interpreted as 0 meaning no relationship, $>+0.30$ as a weak positive relationship, $>+0.50$ as a moderate positive relationship, $>+0.70$ as a strong positive relationship and +1.0 as a perfect positive relationship. To assess agreement in tumor grading (categorical data) between EUS-guided samples and surgical resection histology, Cohen's Kappa was calculated. Kappa values were interpreted as $<0$ indicating no agreement, 0 to 0.20 as poor, 0.21 to 0.40 as fair, 0.41 to 0.60 as moderate, 0.61 to 0.80 as good and 0.81 to 1 as almost perfect agreement. Subgroup analysis was performed for solid lesions, lesions $<2 \mathrm{~cm}$ and different needle types. A $P$ value $<0.05$ was considered statistically significant. Data were analyzed using MedCalc 11.2.1.0 (MedCalc Software, Ostend, Belgium).

\section{Ethical approval}

As per United Kingdom National Health Service research ethics guidance, ethical approval from an institutional review body was not required for this study. Institutional authorization to hold a prospective patient database for use for quality improvement was obtained. Written informed consent was obtained from all patients prior to the procedure.

\section{Results}

One hundred sixty-four patients were diagnosed with PanNET in our center over the study period of whom 57 underwent surgical resection (mean age 55.6, 30 males, mean size $28.3 \mathrm{~mm}$ (standard error \pm 2.3 ). Twenty-two lesions were located in the head, 10 lesions in the body and 25 lesions in the tail of the pancreas with no significant difference in size between the locations $(P=0.13) .41$ were solid, eight cystic and eight mixed morphology. Surgical procedures included distal pancreatectomy with splenectomy $(n=25)$, spleen-preserving distal pancreatectomy $(n=9)$, Whipples' procedure $(n=8)$, enucleation $(n=6)$, pylorus-preserving pancreaticoduodenectomy $(n=5)$, surgical bypass and biopsy $(n=2)$, duodenectomy $(n=1)$ and distal pancreatectomy with splenectomy, sleeve gastrectomy, left nephrectomy, left adrenalectomy, splenic flexure colectomy and diaphragm resection $(n=1)$. On surgical histology 33 lesions were grade 1, 22 were grade 2, one was grade 3 and one was mixed neuroendocrine-acinar.

Thirty-five lesions underwent FNA which was performed using 22-gauge needles in 24 cases, 25-gauge needles in four cases and not stated in seven cases. Twenty-six lesions underwent FNB (4 lesions underwent both FNA and FNB) of which 12 were by Procore needles and 14 usiing Sharkcore needle. For Procore, a 22-gauge needle was used in six cases, 25 gauge needle in five cases and combined 25 and 19 gauge in one case. For Sharkcore, a 22-gauge needle was used in 11 cases and a 25 gauge needle in three cases. - Table 1 shows the demographics of those undergoing EUS sampling with the only significant difference found in distribution of lesion morphology between the FNA and FNB groups. This was to be expected as an FNB needle would not be used for the puncture of a cystic lesion.

Both cytology and histopathology, confirmed a diagnosis of PanNET using morphological features and this is demonstrated in Fig. 1. However, only 23 of 35 FNA samples (66\%) were able to report a Ki67 PI and grade compared to all 26 FNB samples $(100 \%)(P=0.0006)$. For those with reportable values at EUS-FNA, the correlation of Ki67 PI compared to surgical resection histology was poor $(r=-0.08,95 \%$ confidence interval $-0.50-0.38, P=0.74)$. For FNB samples, the correlation of Ki67 PI with surgical histology was moderate $(r=0.65$, $95 \%$ confidence interval $0.35-0.83, P=0.0004)$. With respect to tumor grading, FNA samples showed a poor correlation with a kappa value of 0.026 whereas FNB samples showed a moderate correlation with a kappa value of 0.474 . Table 2 shows the mismatch in categorization for both FNA and FNB samples. Of the 23 FNA samples with a reported grading, 22 were grade 1 on cytology of which seven of 22 were grade 2 on surgical resection and one was grade 3 on cytology but was grade 1 on surgical resection. Of the 26 FNB samples with a reported grading, 17 were grade 1 at FNB but six of 17 were grade 2 on surgical resection and nine were grade 2 at FNB of which one of nine 
- Table 1 Demographics of PanNETs in those undergoing EUS-FNA and EUS-FNB.

\begin{tabular}{|c|c|c|c|}
\hline Variable & FNA group & FNB group & $P$ \\
\hline $\mathrm{N}$ & 35 & 26 & - \\
\hline Mean age (years) & 55.2 & 56.2 & 0.80 \\
\hline Sex & & & 0.61 \\
\hline - Male & 20 & 13 & \\
\hline - Female & 15 & 13 & \\
\hline Mean lesion size $(\mathrm{mm}+$ /- standard error) & $25.7(3.1)$ & $32.5(3.6)$ & 0.16 \\
\hline Lesion location & & & 0.39 \\
\hline - Head & 11 & 12 & \\
\hline - Body & 6 & 5 & \\
\hline - Tail & 19 & 9 & \\
\hline Lesion morphology & & & 0.015 \\
\hline - Solid & 21 & 23 & \\
\hline - Cystic & 8 & 0 & \\
\hline - Mixed & 6 & 3 & \\
\hline Median number of needles passes (IQR) & $2.5(1.0-3.0)$ & $3.0(2.0-4.0)$ & 0.14 \\
\hline Median number of days from EUS to surgery (IQR) & $79.0(50.5-125.5)$ & $65.5(48.0-99.0)$ & 0.37 \\
\hline
\end{tabular}

was grade 1 on surgical resection. There was no statistically significant difference between FNA and FNB regarding grade 1 mismatch $(P=1.0)$.

\section{Lesions $<2 \mathrm{~cm}$}

Overall there were 25 lesions $<2 \mathrm{~cm}$ of which 19 were sampled by FNA and eight by FNB ( 3 were sampled by both). For FNA samples, the correlation of Ki67 PI compared to surgical resection histology was poor $(r=-0.149,95 \% \mathrm{Cl}-0.66-0.53, P=$ 0.75). For FNB samples, the correlation of Ki67 PI compared to surgical resection histology was moderate $(r=0.57,95 \% \mathrm{Cl}-$ $0.23-0.91, P=0.14)$. With respect to tumor grading, FNA samples showed a poor correlation with a kappa value of 0.003 and FNB samples showed a good correlation with a kappa of 0.72 . Of the 11 of 19 FNA samples with a reported grading, 10 were grade 1 and one was grade 3 on cytology but on surgical resection histology 17 were grade 1 , two were grade 2 and none were grade 3 . Of the eight FNB samples, six were grade 1 and two were grade 2 but at surgical resection five were grade 1 and three were grade 2 .

\section{Solid PanNETs}

When cystic and mixed cystic/solid PanNETs were excluded, this left 41 pure solid lesions of which 21 were obtained using FNA and 23 using FNB ( 3 lesions were sampled using both techniques). There were no significant differences in mean size (FNA $27.5 \mathrm{~mm}$ vs. FNB $32.8 \mathrm{~mm}, P=0.38)$, number of needle passes (3.0 vs. $3.0, P=0.91$ ) or lesion location $(P=0.25)$. Ki67 PI could be reported on 14 of 21 FNA samples compared to all 23 FNB samples $(P=0.003)$. For FNA samples, the correlation of Ki67 PI compared to surgical resection histology was poor $(r=-0.102$, $95 \%$ confidence interval $-0.66-0.53, P=0.77$ ). For FNB samples, the correlation of Ki67 PI with surgical resection histology was moderate $(r=0.641,95 \%$ confidence interval $0.31-0.83, P$ $=0.001)$. With respect to tumor grading, FNA samples showed a poor correlation with a kappa value of 0.044 and FNB samples showed a moderate correlation with a kappa of 0.58 . Of the 14 FNA samples with a reported grading, all were grade 1 on cytology but six of 14 were grade 2 on surgical resection histology. Of the 23 FNB samples with a reported grading, 15 were grade 1 and eight grade 2 . At surgical resection five of 15 which were grade 1 at EUS-FNB were found to be grade 2 but all of those that were grade 2 at EUS-FNB were confirmed as grade 2 at surgical resection. There was no statistically significant difference between FNA and FNB regarding grade 1 mismatch $(P=0.71)$.

\section{FNB needle subgroup analysis}

Ki67 PI and grading could be reported on all FNB samples irrespective of the two needle types (Procore and Sharkcore). Twelve samples had been obtained using the Procore needle (reverse bevel) and 14 samples obtained using the Sharkcore needle (Fork tip). For Procore samples, the correlation of Ki67 PI with surgical resection histology was moderate $(r=0.521$, $95 \%$ confidence interval-0.07-0.84, $P=0.08$ ). For Sharkcore samples, the correlation of Ki67 PI with surgical resection histology was good $(r=0.788,95 \%$ confidence interval $0.42-$ 


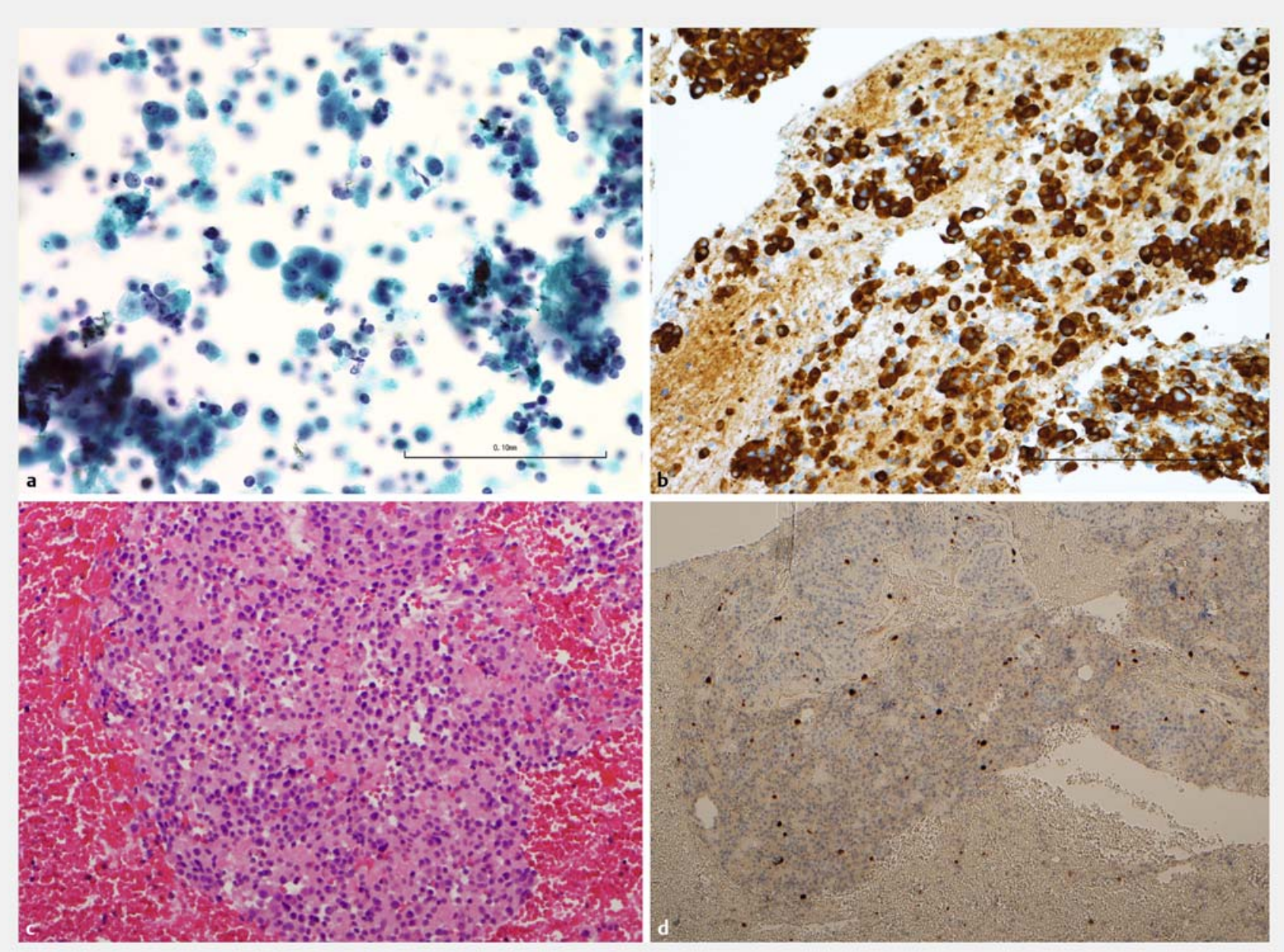

- Fig. 1 Examples of EUS-FNA and EUS-FNB samples of PanNETs. a FNA sample Papanicolaou x40 magnification. b FNA sample synaptophysin staining x40 magnification. c FNB sample H\&E x40 magnification. d FNB sample synaptophysin staining high magnification.

- Table 2 EUS-FNA and EUS-FNB sample tumor grading compared to surgical resection histology.

\begin{tabular}{|l|l|l|l|l|}
\hline & \multicolumn{4}{|c|}{ Surgical resection sample } \\
\hline & Grade 1 & Grade 2 & Grade 3 & Unable \\
\hline FNA grade 1 & 15 & 7 & 0 & 0 \\
\hline FNB grade 1 & 11 & 6 & 0 & 0 \\
\hline FNA grade 2 & 0 & 0 & 0 & 0 \\
\hline FNB grade 2 & 1 & 8 & 0 & 0 \\
\hline FNA grade 3 & 1 & 0 & 0 & 0 \\
\hline FNB grade 3 & 0 & 0 & 0 & 0 \\
\hline FNA unable & 8 & 3 & 1 & 0 \\
\hline FNB unable & 0 & 0 & 0 & 0 \\
\hline
\end{tabular}

EUS-FNA, endoscopic ultrasound-guided fine-needle aspiration; EUS-FNB, endoscopic ultrasound-guided fine-needle biopsy
0.93, $P=0.0013)$. With respect to tumor grading, Procore samples showed a moderate correlation with a kappa of 0.47 and Sharkcore samples similarly showed a moderate correlation with a kappa of 0.435 .

\section{Discussion}

The current study shows that FNB needles are significantly more likely to provide sufficient pathological material that can be used to characterize PanNETs. Furthermore, when compared to surgical resection histology, FNB samples had a closer correlation with respect to Ki67 PI and tumor grading than FNA samples. This difference was maintained when cystic PanNETs were excluded. This subgroup analysis was performed as there was a significant difference in the distribution of lesion morphology when comparing FNA to FNB. Crucially, in lesions $<2 \mathrm{~cm}$, FNB samples correlated significantly better than FNA samples but was only moderate compared to the final surgical resection sample. When looking at the different subtypes of FNB needles, Sharkcore samples appeared to have the closest correlation of Ki67 PI to surgical resection histology but no difference in 
agreement with overall grading. This reflects the closer correlation of the Ki67 PI in Sharkcore samples to the final surgical histology compared to the other needle type. However, the range of Ki67 PI for grade 2 is large (e. g. $2 \%$ to $20 \%$ ) and therefore if an endoscopic sample was reported as $2.5 \%$ and the surgical sample $18 \%$ this would be grade 2 for both samples. Clinically, whilst the grade is helpful, a more precise Ki67 PI supports clinical decision-making.

Both FNA and FNB appeared to under-grade lesions that were found to be grade 2 on surgical histology. In fact, the vast majority of FNA samples were reported as grade 1, none as grade 2 and one as grade 3. Several of those classified as grade 1 were upgraded at resection and the grade 3 sample was found to be grade 1 at surgery. In the FNB group there were more confident classifications into grade 2 although there was still a proportion classified as grade 1 that were ultimately grade 2 on surgical resection.

PanNETs are rare but carry a more favorable prognosis when compared to pancreatic adenocarcinoma [1,2]. Prognosis and management in PanNETs is closely linked to Ki67 PI and tumor grade, therefore, accurate sampling of such lesions is essential [4-6]. Many PanNETs are sampled pre-peratively using EUS but the accuracy of EUS- FNA has been shown to vary [9-13]. The recent addition of FNB needles to the endosonographers' armamentarium may improve the diagnostic accuracy $[18,20]$ and tumor grading but there is a paucity of data concerning their use for PanNETs. EUS has become the modality of choice for assessment and sampling of PanNETs with a reported sensitivity of up to $80 \%$ to $90 \%$ [9]. Previous studies have shown that EUS-FNA samples can be used to report Ki67 PI but the correlation with surgical resection histology is unclear. Surgical resection histology was not the gold standard in all of these studies and many were small $(n<20)[10,11,13-17]$. Two recent studies have shown that cytology tends to underestimate grade 2 lesions and the concordance was affected specimen cellularity $[16,17]$.

One recent study compared FNA to FNB in patients with suspected PanNETs $(n=20)$ which showed that FNB required fewer passes to obtain a diagnosis. There was no reporting of KI67 PI or grading or comparison with surgical histology in this study [22].

There are limitations to the current study. Primarily, this is a retrospective analysis in patients with a positive diagnosis. Many patients referred to our unit will have had a single sampling either by FNA or FNB but no other tissue samples taken to make comparison, mainly due to advanced stage on imaging negating the need. There were also a number of patients who proceeded straight to surgery without preoperative biopsy and there is a possibility that this contributed some bias. Both of these factors, however, would be expected to affect both FNA and FNB equally. During the study period the classification scheme for grading PanNETs changed [24,25]. However, to minimize the bias this might have caused, each sample, whether endoscopic or surgical, was allocated according to the grading system used at that time period. This meant that allocation to a grade was consistent for each lesion. There were also very few lesions that had undergone simultaneous FNA and FNB, therefore, the differences between the groups may have been affected by this. Ideally, a prospective study using both FNA and FNB needles in this group of patients would be required to determine which is optimal for sampling. The most recent guidelines for assessing PanNETs recommend a count of 500 to 2000 cells in the area of highest labelling in intact tumor tissue to provide the most accurate assessment of Ki67 [24,25]. One of the difficulties of EUS-guided samples is that only a small part of the lesion is sampled and often fewer than 500 cells may be available for counting. This means that there is not a representative "hotspot" to assess, therefore, the lesion may be under-graded. Furthermore, current recommendations are to photograph the "hotspot" and then manually count 2000 cells. This practice was not consistently used throughout the study period. Future studies should set a minimum number of cells present before Ki67 assessment can be performed, recording of magnification used, intensity of staining seen and which aids were used to facilitate cell counts. These factors apply to both histology and cytology samples and were not standardized during the study period. Also, with respect to cytology samples particularly, cellular dissociation may affect assessment of tumor and non-tumor cells following staining, which is especially seen in liquid-based cytology samples. Cell blocks may mitigate this effect, however, samples were reported overall rather than by the individual components. There was a tendency to perform more passes with the FNB needle (3.0 vs 2.5) which may have affected the study outcome despite not being statistically significantly different. In a study with larger numbers this may have contributed to improved pathological samples. Furthermore, different needle calibers were used depending upon endosonographer preference, which may have also had an effect on tissue acquisition [26]. Also, because our study was conducted over several years, it is possible that either the endosonographers or pathologists have moved up the learning curve and therefore affected the results. This may be relevant as FNB needles have only become available in the last few years. Finally, the majority of lesions were grade 1 or 2, therefore, the accuracy of FNA or FNB in grade 3 lesions cannot be commented upon in this study. However, most grade 3 lesions would be put forward for surgical resection or systemic therapy and the area of most interest being whether it is safe to truly classify a lesion as grade 1 using solely endoscopic sampling. Strengths of the current study include representation of one of the largest endoscopic series in patients presenting with PanNETs with FNA and FNB from a high-volume center with experienced endosonographers and pathologists. Also, EUS-guided samples KI67 PI and grade were compared to surgical resection histology as the gold standard unlike in most previous studies $[10,11$, $13,14,16,17]$.

\section{Conclusion}

In summary, in the current study we have shown that FNB is superior to FNA for accurate characterization of grading of PanNET lesions with the best correlation to surgical resection histology seen using the Sharkcore needle. A randomized, controlled, multicenter, crossover trial in which lesions are sam- 
pled using both FNA and FNB with blinding of the pathologists will be required to fully elucidate which needle type is optimal.

\section{Competing interests}

Drs, Leeds, Nayar, Johnson, Haugk, Darne and Oppong have all received investigator-initiated trial research funding from Medtronic. Drs. Leeds and Nayar have consulted for Medtronic.

\section{References}

[1] Yao JC, Hassan M, Phan A et al. One hundred years after "carcinoid": epidemiology of and prognostic factors for neuroendocrine tumors in 35,825 cases in the United States. J Clin Oncol 2008; 26: 3063-3072

[2] Metz DC, Jensen RT. Gastrointestinal neuroendocrine tumours: pancreatic endocrine tumours. Gastroenterology 2008; 135: 1469-1492

[3] Fitzgerald TL, Hickner Z], Schmitz M et al. Changing incidence of pancreatic neoplasms: a 16-year review of statewide tumor registry. Pancreas 2008; 37: 134-138

[4] Rindi G, Petrone G, Inzani F. The 2010 WHO classification of digestive neuroendocrine neoplasms: a critical appraisal four years after its introduction. Endocr Pathol 2014; 25: 186 - 192

[5] Falconi M, Bartsch DK, Eriksson B et al. ENETS Consensus Guidelines for the management of patients with digestive neuroendocrine neoplasms of the digestive system: well-differentiated pancreatic nonfunctioning tumors. Neuroendocrinology 2012; 95: 120 - 134

[6] Rindi G, Falconi M, Klersy C et al. TNM staging of neoplasms of the endocrine pancreas: results from a large international cohort study. J Natl Cancer Inst 2012; 104: 764 - 777

[7] Ganetsky A, Bhatt V. Gastroenteropancreatic neuroendocrine tumors: update on therapeutics. Ann Pharmacother 2012; 46: 851 - 862

[8] Vilmann P, Jacobsen GK, Henriksen FW et al. Endoscopic ultrasonography with guided fine needle aspiration biopsy in pancreatic disease. Gastrointest Endosc 1992; 38: $172-173$

[9] Attili F, Capurso G, Vanella G et al. Diagnostic and therapeutic role of endoscopy in gastroenteropancreatic neuroendocrine neoplasms. Dig Liver Dis 2014; 46: 9-17

[10] Figueiredo FA, Giovannini M, Monges $G$ et al. EUSFNA predicts 5-year survival in pancreatic endocrine tumors. Gastrointest Endosc 2009; 70: $907-914$

[11] Hasegawa T, Yamao K, Hijioka S et al. Evaluation of Ki-67 index in EUSFNA specimens for the assessment of malignancy risk in pancreatic neuroendocrine tumors. Endoscopy 2014; 46: 32 - 38
[12] Figueiredo FA, Giovannini M, Monges $G$ et al. Pancreatic endocrine tumors: a large single-center experience. Pancreas 2009; 38: 936 940

[13] Piani C, Franchi GM, Cappelletti C et al. Cytological Ki-67 in pancreatic endocrine tumours: an opportunity for pre-operative grading. Endocr Relat Cancer 2008; 15: 175-181

[14] Chatzipantelis $P$, Konstantinou P, Kaklamanos $M$ et al. The role of cytomorphology and proliferative activity in predicting biologic behavior of pancreatic neuroendocrine tumors: a study by endoscopic ultrasound-guided fine needle aspiration cytology. Cancer 2009; 117: 211-216

[15] Alexiev BA, Darwin PE, Goloubeva O et al. Proliferative rate in endoscopic ultrasound fine-needle aspiration of pancreatic endocrine tumors: correlation with clinical behavior. Cancer 2009; 117: 40 - 45

[16] Weynand B, Borbath I, Bernard V et al. Pancreatic neuroendocrine tumour grading on endoscopic ultrasoundguided fine needle aspiration: high reproducibility and inter-observer agreement of the Ki-67 labelling index. Cytopathology 2014; 25: 389-395

[17] Larghi A, Capurso G, Carnuccio A et al. Ki-67 grading of nonfunctioning pancreatic neuroendocrine tumors on histologic samples obtained by EUS-guided fine-needle tissue acquisition: a prospective study. Gastrointest Endosc 2012; 76: 570 - 577

[18] Nayar MK, Paranandi B, Dawwas MF et al. Comparison of the diagnostic performance of 2 core biopsy needles for EUS-guided tissue acquisition from solid pancreatic lesions. Gastrointest Endosc 2017; 85: $1017-1024$

[19] DiMaio C], Kolb JM, Benias PC et al. Initial experience with a novel EUSguided core biopsy needle (SharkCore): results of a large North American multicenter study. Endosc Int Open 2016; 4: E974 - E979

[20] Kandel P, Tranesh G, Nassar A et al. EUS-guided fine needle biopsy sampling using a novel fork-tip needle: a case-control study. Gastrointest Endosc 2016; 84: 1034 - 1039

[21] Bang JY, Hawes R, Varadarajulu S. A meta-analysis comparing ProCore and standard fine-needle aspiration needles for endoscopic ultrasound-guided tissue acquisition. Endoscopy 2016; 48: 339-349

[22] Witt BL, Factor RE, Chadwick BE et al. Evaluation of the SharkCore ${ }^{\circledR}$ needle for EUS-guided core biopsy of pancreatic neuroendocrine tumors. Endosc Ultrasound 2018; 7: 323-328

[23] Nayar MK, Chatterjee S, Wadehra V et al. Does on-site adequacy assessment by cytotechnologists improve results of EUS guided FNA of solid pancreaticobiliary lesions? JOP 2013; 14: $44-49$

[24] Bosman FT, Carneiro F, Hruban RH et al. WHO classification of tumours of the digestive system.4th edition France: IARC; 2010

[25] Stephenson T], Cross SS, Chetty R. Dataset for neuroendocrine tumours of the gastrointestinal tract including pancreas.3rd edn. London: The Royal College of Pathologists; 2012

[26] Kandel P, Wallace MB. Advanced EUS guided tissue acquisition methods for pancreatic cancer. Cancers 2018; 10: pii E54 